\title{
Comment on: Botulinum Toxin Type A Overdoses: Analysis of the FDA Adverse Event Reporting System Database
}

\author{
Philippe Picaut $^{1}$ (D)
}

Published online: 3 November 2018

(c) The Author(s) 2018

\section{Dear Editor,}

The recent publication by Kazerooni and Armstrong [1] investigated overdose with botulinum neurotoxin type-A (BoNT-A) treatment using data from the US Food and Drug Administration's (FDA) Adverse Event Reporting System (FAERS). Although the authors highlighted an important topic in overdose, the methodology used does not account for limitations of the FAERS database, and as such the results and conclusions are inaccurate, with potential to erroneously impact standard clinical practice and unduly alarm physicians.

The researchers used the FAERS database to perform a comparison of overdose and associated adverse events between the BoNT-A products abobotulinumtoxinA (Dysport $^{\circledR}$ ), onabotulinumtoxin $A\left(\right.$ Botox $\left.^{\circledR}\right)$, and incobotulinumtoxinA $\left(\mathrm{Xeomin}^{\circledR}\right)$. Although this database does contain information related to overdose and adverse events [2], important disclaimers regarding its limitations and appropriate use are stipulated on the FDA website, including but not limited to [3]:

- Lack of any verification of reports.

- Potential report duplication.

- Lack of causality between product and event.

A statement on the FAERS website clearly notes that: "Rates of occurrence cannot be established with reports"

This comment refers to the article available at https://doi. org/10.1007/s40261-018-0668-7.

An authors' reply to this comment is available at https://doi. org/10.1007/s40261-018-0724-3.

Philippe Picaut

philippe.picaut@ipsen.com

1 Ipsen Bioscience, Inc., 650 East Kendall Street, Cambridge, MA 02142, USA
[3]. The analyses performed by Kazerooni and Armstrong contravene these disclaimers, rendering the comparative assessment inaccurate. Upon entering the FAERS database, all users are required to acknowledge the following statement: "FAERS data alone cannot be used to establish rates of events, to evaluate a change in event rates over time or to compare event rates between drug products. The number of reports cannot be interpreted or used in isolation to reach conclusions about the existence, severity, or frequency of problems associated with drug products" [4]. In addition to this fundamental challenge to the reported analyses, I noted several inaccuracies in the paper.

In the retrospective review of FAERS data, Kazerooni and Armstrong aimed to determine the incidence of overdose reports for each of the BoNT-A products. The authors did not set any objective parameters for overdose cases to aid the analysis. Rather, overdose was defined based on the subjective reporting of an overdose event to the FAERS database by "Healthcare professionals, consumers, [or] manufacturers" [2]. Because no information about doses injected or indication treated, as per the local labels, are included in the FAERs database, it is not possible to perform a clinically meaningful interpretation of these overdose cases and associated adverse events. Potency units, dosing, and reconstitution are different and are not interchangeable between BoNT-A products [5-7]. As dosages per muscle are numerically higher for abobotulinumtoxinA compared with the other BoNT-A products, this may have led reporting individuals to believe that an overdose was given or received when making reports to the FAERS database.

A subgroup analysis was performed in the Kazerooni article [1] to investigate overdose and the incidence of eyelid ptosis, selected "because cosmetic use was the most common indication and distant spread of toxin, consistent with a possible overdose, is a boxed warning for all three products" [1]. I consider this a potentially misleading medical statement and analysis because this was performed solely on cosmetic cases. Botulinum neurotoxin type-A cosmetic use is 
predominantly for indications for facial lines, such as glabellar lines or lateral canthal lines, where BoNT-A is injected in anatomical proximity to the eyelids. Therefore, eyelid ptosis is not considered an effect of distant spread for aesthetic indications and should rather be considered as a local effect. I note that the results reported in Kazerooni and Armstrong differ from, and contradict, the reported incidence of eyelid ptosis included in the US prescribing information for each BoNT-A product, based on data from clinical trials with rigorous adverse-event reporting systems. For glabellar lines indications, at approved doses, the incidence of eyelid ptosis is comparable across products (abobotulinumtoxinA 2\%, onabotulinumtoxinA 3\%, and incobotulinumtoxinA $0.2 \%$ ) [5-7].

In conclusion, I assert that the analyses presented in the paper by Kazerooni and Armstrong are inaccurate from conception by inappropriate use of the FAERS database, which has limitations owing to data input constraints that are acknowledged by the FDA. The FAERS website includes disclaimers directly advising against the type of analysis that was performed. Given the importance of providing patients and physicians with correct and accurate safety information, I object to the analyses published in the Kazerooni and Armstrong article and suggest that the article should be retracted.

\section{Compliance with Ethical Standards}

Funding Submission support was provided by Watermeadow Medical, an Ashfield Company, part of UDG Healthcare plc, funded by Ipsen.

Conflict of interest Philippe Picaut is an employee of Ipsen.

Open Access This article is distributed under the terms of the Creative Commons Attribution-NonCommercial 4.0 International License (http://creativecommons.org/licenses/by-nc/4.0/), which permits any noncommercial use, distribution, and reproduction in any medium, provided you give appropriate credit to the original author(s) and the source, provide a link to the Creative Commons license, and indicate if changes were made.

\section{References}

1. Kazerooni R, Armstrong EP. Botulinum toxin type A overdoses: analysis of the FDA Adverse Event Reporting System database. Clin Drug Investig. 2018;38:867-72.

2. US Food and Drug Administration. Questions and answers on FDA's Adverse Event Reporting System (FAERS). https://www. fda.gov/Drugs/GuidanceComplianceRegulatoryInformation/ Surveillance/AdverseDrugEffects/default.htm. Accessed $11 \mathrm{Jul}$ 2018.

3. US Food and Drug Administration. FDA Adverse Event Reporting System (FAERS) public dashboard. https://www.fda.gov/drugs /guidancecomplianceregulatoryinformation/surveillance/adver sedrugeffects/ucm070093.htm. Accessed 27 Jun 2018.

4. US Food and Drug Administration. FDA Adverse Events Reporting System (FAERS) public dashboard disclaimer. https://fis. fda.gov/sense/app/d10be6bb-494e-4cd2-82e4-0135608ddc13/ sheet/7a47a261-d58b-4203-a8aa-6d3021737452/state/analysis. Accessed 19 Oct 2018.

5. Dysport ${ }^{\circledR}$ (abobotulinumtoxinA) for injection: US prescribing information. Basking Ridge, NJ: Ipsen Biopharmaceuticals, Inc. 2016. https://www.accessdata.fda.gov/drugsatfda_docs/label /2016/125274s107lbl.pdf. Accessed 19 Oct 2018.

6. Botox ${ }^{\circledR}$ (onabotulinumtoxinA): US prescribing information. Irvine, CA, USA: Allergan. 2017. https://www.allergan.com/asset s/pdf/botox_cosmetic_pi.pdf. Accessed 19 Oct 2018.

7. Xeomin ${ }^{\circledR}$ (incobotulinumtoxinA): US prescribing information. Frankfurt, Germany: Merz Pharmaceuticals GmbH. 2015. https:// www.xeominaesthetic.com/wp-content/uploads/2017/09/EM004 51-02-Electronic-PI-EM00674-02-Med-Guide.pdf. Accessed 19 Oct 2018. 